\title{
EVALUATION OF DROUGHT TOLERANCE AND HETEROSISE OF SOME ROOT CHARACTERS IN RICE (Oryza sativa $L$.)
}

\author{
Nessreen N. Bassuony and G.B. Anis \\ Rice Research Section, Sakha, Kafrelshiekh ,Field Crops \\ Research Institute, A.R.C. ,El-Giza, Egypt.
}

\begin{abstract}
The present investigation was conducted at the Rice Research and Training Center (RRTC),Sakha, Kafrelsheikh, Egypt, during 2014 and 2015 growing seasons to evaluate some root characters of some rice genotypes for drought and well-watered conditions and determination of heterosis of some root characters in rice (Oryza sativa, L.) in relation to drought tolerance.

Preliminary analysis of variance showed that genotypes were differently influenced by sowing conditions. Growth rate $\left(g\right.$ day $\left.^{-1}\right)$ differed significantly among the genotypes studied. The genotype, Nerica4 had much greater growth rate than other genotypes under study.

It was clear that drought stress reduced total root length for all five genotypes. Root volume differentially decreased to a range from 24.53to 19.17 under drought condition. Numbers of total roots were significantly different under the two sowing conditions. Under well watered, the number of roots increased as compared with drought condition. In addition, analysis of variance showed that root dry weight was higher at the well watered compared to drought, meantime root to shoot ratio decreased under drought. The highest values were recorded under well watered condition.

The magnitude of heterosis manifested over mid parent and better parents are presented. Highly significant estimates of heterosis as a deviation from mid parent and better parent were exhibited in all studied crosses for growth rate $\left(\mathrm{g}\right.$ day $\left.^{-1}\right)$ and root length $(\mathrm{cm})$ under normal and drought conditions crosses for growth rate $\left(\mathrm{g} \mathrm{day}^{-1}\right)$ and root length $(\mathrm{cm})$ are desirable for drought tolerance in rice.
\end{abstract}

\section{INTRODUCTION}

Water shortages are responsible for the greatest crop losses around the world and are expected to worsen, heightening international interest in crop drought tolerance. About $67 \%$ of crop losses over the last 50 years have been due to drought and more frequent occurrences of water shortages are expected due to climate projections and increasing competition for water among urban, industrial, and agricultural demand (IPCC, 2012; Harovon Mogel, 2013).Geneticists and breeders are in position to make strides in breeding plants for better yield. Drought tolerance is most desirable as the maintenance of crop productivity under water deficient conditions which can be accomplished in a variety of ways, including drought 
avoidance or desiccation prevention, potentially in combination, through matching crop water use with water availability, and recovery of growth following rewetting (Passioura, 2012).

Root system plays an important role under drought conditions. The nature and extent of root characteristics are considered to be major factors affecting plant response to water stress. Regardless of the ecosystem where rice breeding is aimed, researchers look toward understanding the role of roots for improving nutrient and water acquisition and increasing grain yield. There are significant differences reported for root thickness, depth, and root mass among rice cultivars and there is documented genetic variation for root morphological traits in response to drought (Kondo et al., 2003and Gowda et al., 2011). However, this variation and how it influences the crop's root function for water uptake under drought remains to be fully understood (Gowda et al., 2011). Various screening methods used to identify root traits associated with drought tolerance in rice germplasm. Root dry mass and length, commonly assessed by direct evaluation, is a good predictor of yield in rice (Beyrouty, 2002 and Fageria and Moreira, 2011).

A deep root system could improve the adaptation of rice during drought through greater capacity for water extraction, thus maintaining high plant leaf water statue, Cleber et al,. (2013).

The use of managed drought stress, where it can be imposed at specific periods, has been shown to increase the heritability of yield under stress to values similar to those obtained for yield in wellwatered conditions. It has now been demonstrated that droughttolerant upland rice can be bred by directly selecting for yield in stress environments (Bernier et al., 2008).

The objective of this study was to evaluate the drought tolerance of some genotypes of drought rice with broad genetic diversity grown under water stressed and well watered conditions as well as determination of heterosis of some root characters in rice (Oryza sativa, L.) in relation to drought tolerance.

\section{MATERIALS AND METHODS}

The present investigation was conducted at experimental farm of the Rice Research and Training Center (RRTC),Sakha, Kafrelshiekh, Egypt, during 2014 and 2015 growing seasons to evaluate some root characters of some rice genotypes under drought and well-watered conditions and determination of heterosis of some root characters in rice (Oryza sativa, L.) in relation to drought tolerance.

The experimental materials consisted of Five rice genotypes; three tolerant genotypes (Nerica4, Wab 1573 and BG304), one moderate tolerant genotype (Giza 179) and one sensitive genotype 
(Sakha 102). Origin and main characters of the five genotypes used as parents in the studied crosses are presented in (Table, 1).

Table 1: Origin and main characters of the five genotypes used as parents in the studied pair crosses

\begin{tabular}{|l|c|c|c|c|c|}
\hline No & Genotype & Origin- Parentage & $\begin{array}{l}\text { Grain } \\
\text { shape }\end{array}$ & $\begin{array}{l}\text { Variety } \\
\text { group }\end{array}$ & $\begin{array}{l}\text { Drought } \\
\text { tolerant }\end{array}$ \\
\hline 1 & Nerica 4 & $\begin{array}{c}\text { Africa Rice } \\
\text { TOG681-3/IR 64 }\end{array}$ & long & Indica & Tolerant \\
\hline 2 & Wab 1573 & Warda & long & Indica & Tolerant \\
\hline 3 & BG304 & Sirilanka & long & Indica & Tolerant \\
\hline 4 & Giza 179 & $\begin{array}{c}\text { Egypt } \\
\text { GZ1368-S-5-4/IRAT } \\
112\end{array}$ & Short & $\begin{array}{c}\text { Japonica- } \\
\text { Indica }\end{array}$ & Moderate \\
\hline 5 & Sakha 102 & $\begin{array}{c}\text { Egypt } \\
\text { (GZ4096-7-1/Giza177) }\end{array}$ & Short & Japonica & Sensitive \\
\hline
\end{tabular}

In 2014 two experiments in a randomized complete block design (RCBD) were conducted under normal and drought conditions with three replication consisted of all utilized genotypes. Besides at flowering, a crossing program was carried out to produce $F 1$ hybrid seeds of three crosses namely; I- Nerica4 (tolerant) $x$ Wab 1573(tolerant).II- BG 1573 (tolerant) x Giza 179 (moderate) III- Nerica4 (tolerant)xSakha 102(sensitive). The F1 of the resultant three crosses along with their parents were harvested.

In 2015 season, parents and half of $\mathrm{F} 1$ hybrid seeds of the three crosses were sown under drought conditions however, the other half and the same parents were sown under normal condition. The two experiments were raised in RCBD with three replications. Each replicate contained 5 rows of parents and three crosses (F1) with a spacing of $20 \times 20 \mathrm{~cm}$. Single seedling was transplanted per hill for each. In all growing seasons of the study, all cultural practices such as field preparation, sowing, and fertilizers were applied as recommended.

Samples for roots characters study were taken every ten days after transplanting to maximum tillering stage.

Heterosis effects were estimated as deviation of mid-parent (M.P) and better parent (B.P) according to Mather and Jinks (1971) as follow:

Heterosis over the mid parent $=\frac{F^{-1}-M P^{-}}{M P^{-}} x 100$

$\mathrm{S} . \mathrm{E}\left(\mathrm{F} 1-M P^{-}\right)=(3 \mathrm{Me} / 2 \mathrm{r}) 1 / 2$

Heterosis over the better- parent $=\frac{F^{-1}-B^{-} P^{-}}{B^{-} P^{-}} \times 100$

$\mathrm{S} . \mathrm{E}\left(\mathrm{F} 1-\mathrm{BP}^{-}\right)=(2 \mathrm{Me} / \mathrm{r}) 1^{2}$ 
Where. $\mathrm{Ne}=$ error mean squares for parents and $\mathrm{F} 1$ in an individual environment; $\mathrm{MP}^{-}=$mean mid - parent value $=(\mathrm{P} 1+\mathrm{P} 2) / 2 ; \mathrm{P} 1=$ mean performance of parent one; $\mathrm{P} 2=$ mean performance of parent two; $\mathrm{BP}^{-}=$mean of better- parent value; r-number of replication.

The following data for the root characters are taken:

\section{1- Relative growth rate (g/day):}

Each sample was replicated three times. Relative growth rate was calculated using root dry weight, Sestak et al., (1971).by the formula

Growth rate $\left(\mathrm{g}_{\text {day }}{ }^{-1}\right)=\frac{\operatorname{In} \mathrm{W}_{2}-\mathrm{In} \mathrm{W}_{1}}{\mathrm{tz}-\mathrm{t} 1}$

Where $\mathrm{W} 1$ and $\mathrm{W} 2$ are weight of roots at time t1and $\mathrm{t} 2$.

At maximum tillering stage, various physiological characteristics of shoot and root were measured. At the end of this stage, twenty plants from each plot were pouled, and shoots were separated from roots. Roots were washed free of soil. Length of all roots in each plot was measured with an image analysis system.

\section{2- Root length (cm):}

The root length of plant as average of twenty plants from each plot was measured from the base of roots until the end of white root.

\section{3-Root numbers:}

Three plants per plot were completely dug out during the tillering stage. After being washed carefully, the underground parts were placed on coordinate paper and the number of roots per plant at $30 \mathrm{~cm}$ length was calculated, respectively. These roots were washed again. After removing impurities and dead roots, and then roots were dried and weighed. The mean weight of a total of 27 plants from three plots with three plants per plot during tillering stage was used for comparison.

4 -Root dry weight (g plant $\left.{ }^{-1}\right)$ : Shoots and roots were oven-dried at $85^{\circ} \mathrm{C}$ for $72 \mathrm{~h}$ until no further weight loss occurred. Root dry weight was determined, the root dry weight of plant as average of twenty roots of each plot collected, , carefully separated, washed, oven dried and then root dry weight was recorded in g plant $^{-1}$.

\section{5-Root / Shoot ratio (R/S ratio):}

Root / Shoot ratio was calculated using the following equation:

$\mathrm{R} / \mathrm{S}$ ratio $(\%)=\frac{\text { Root dry weight (g plant }-1)}{\text { Shoot dry weight (g plant-1) }} \times 100$

After samples had been oven-dried at $85^{\circ} \mathrm{C}$ for $72 \mathrm{~h}$, roots and shoot were weighed per plant.

\section{6-Specific root lengths:}

Ratio of root length to root weight were calculated. An elevated specific root length indicates an efficient fine-root proliferation for a given allocation of assimilates to roots- $(S R L=$ root length per unit root 
weight) was calculated. Dry shoots and roots were then ground with a cyclone sample mill according to the method of Kato and Okami(2011). The phenotypic correlation coefficient was performed according to the procedure of Dewey and Lu (1959).

All collected data were subjected to analysis of variance according to Gomez and Gomez (1984). Treatments means were compared by Duncan's multiple range test (Duncan, 1955). All statistical analysis was performed using variance technique by means of "MSTAT" computer soft war package.

\section{RESULTS AND DISCUSSION}

The analysis of variance for growth rate $\left(\mathrm{g} / \mathrm{day}^{-1}\right)$, root length $(\mathrm{cm})$, root volume $\left(\mathrm{cm}^{3}\right)$ and number of roots per plant characters under study were presented in (Table, 2). Preliminary analysis of variance showed that genotypes were differently influenced by sowing conditions, there was reduction in root growth rate during drought (Siopongco et al, 2005) .Rice varieties differed in their growth rate $(\mathrm{g}$ day $\left.^{-1}\right)($ Table, 2$)$. Growth rate $\left(g_{\text {day }}{ }^{-1}\right)$ differed significantly among the genotypes studied. The genotype, Nerica 4 had much greater growth rate than other genotypes under study and there was no significant differences among Wab 1573, BG 304 and Nerica 4. Sakha 102 had the fewer growth rate $\left(\mathrm{g} \mathrm{day}^{-1}\right)$ under both well watered and drought conditions.

The results indicated that the maximum root length was higher at maximum tillering stage of growth under well watered condition. It was clear that drought stress reduced total root length for all five genotypes. Root length differed among genotypes under well-watered and drought conditions, but Nerica 4 was the highest one $(43.67 \mathrm{~cm}$ and $28.13 \mathrm{~cm}$ under well watered and drought conditions, respectively). Root length did not differ among Sakha 102, Giza 179 and Wab 1573 under well-watered conditions. Total root length is strongly related to drought tolerance in rice under upland conditions (Ingram et al., 1994). The Tolerant genotypes had the highest root length than intolerant genotypes, so they could effectively use more water stored at the deeper soil layers. Differences in the root density of deep and shallow rooted plants were found in the soil layers deeper than $20 \mathrm{~cm}$. (Abd Allah, et al 2010).

Root volume of studied genotypes ranged from 43.0 to 24.67

$\mathrm{cm}^{3}$

under well watered and the root volume differentially decreased to a range of 24.53 to 19.17 under drought condition (Table,2). The data showed that the genotypes BG304 and Nerica 4 produced the highest root volume under well-watered, Nerica 4 was the highest root under drought while, the variety, Sakha 102 produced the lowest one under 
drought and well- watered. The Tolerant genotypes had volume of root than intolerant and root volume might be important in water uptake and translocation (Fitter, 1991).

Numbers of total roots were significantly different under the two sowing conditions. Under well watered, the number of roots decreased as compared with drought. The genotypes under study significantly differed

In root number. Maximum root number was recorded by Nerica 4(356.83 and 170.0 under well watered and drought, respectively) the minimum value was found in Sakha 102 (194.0 under well watered and 123.0 under drought).Drought tolerant rice genotypes had fewer numbers of roots, but a higher proportion of the roots were distributed in the lower soil layers below $20 \mathrm{~cm}$. As consequence, assimilate supply to roots of the mother shoot would be reduced, resulting in restricted root growth. Also, even after the tiller has become sustaining, an increased number of tillers/plant would increase mutual shading within the plant. Shading reduces root growth more than shoot growth. Abd Allah, et al, 2010).

Table(2): Influence of well-watered and drought on root growth rate from 21 to 43 days after sowing $\left(\mathrm{g} \mathrm{day}^{-1}\right)$, root length $(\mathrm{cm})$, root volume $\left(\mathrm{cm}^{3}\right)$ and no. of roots/plant $(\mathrm{g})$ below $30 \mathrm{~cm}$ from soil surface of rice genotype at maximum tillering stage

\begin{tabular}{|c|c|c|c|c|}
\hline $\begin{array}{l}\text { Genotypes } \\
\text { Root }\end{array}$ & $\begin{array}{c}\text { growth rate } \\
\left(\text { g day }^{-1}\right)\end{array}$ & $\begin{array}{l}\text { Root length } \\
\text { (cm) }\end{array}$ & $\begin{array}{l}\text { Root volume } \\
\left(\mathrm{cm}^{3}\right)\end{array}$ & $\begin{array}{l}\text { No. of roots } \\
\text { / plant }\end{array}$ \\
\hline \multicolumn{5}{|l|}{ Well-watered } \\
\hline Sakha 102 & $0.105 \mathrm{~b}$ & $23.67 \mathrm{c}$ & $24.67 \mathrm{~b}$ & $194.0 \mathrm{~d}$ \\
\hline Giza 179 & $0.148 \mathrm{~b}$ & $24.00 \mathrm{c}$ & $25.83 \mathrm{~b}$ & $200.0 \mathrm{c}$ \\
\hline Nerica 4 & $0.204 a$ & $43.67 \mathrm{a}$ & $37.00 \mathrm{a}$ & $356.83 a$ \\
\hline Wab 1573 & $0,177 a b$ & $24.16 \mathrm{c}$ & $30.0 \mathrm{ab}$ & $316.67 \mathrm{~b}$ \\
\hline BG 304 & $0.166 a b$ & $31.00 \mathrm{~b}$ & $43.00 \mathrm{a}$ & $216.0 \mathrm{c}$ \\
\hline F-test & & ** & ** & ** \\
\hline \multicolumn{5}{|l|}{ Drought } \\
\hline Sakha 102 & $0.085 \mathrm{~b}$ & $16.33 \mathrm{e}$ & $19.17 \mathrm{c}$ & $123.0 \mathrm{~d}$ \\
\hline Giza 179 & $0.090 \mathrm{~b}$ & $20.00 \mathrm{~d}$ & $20.0 \mathrm{bc}$ & $140.0 \mathrm{c}$ \\
\hline Nerica 4 & $0.188 \mathrm{a}$ & $28.13 \mathrm{a}$ & $24.53 \mathrm{a}$ & $170.0 \mathrm{a}$ \\
\hline Wab 1573 & $0.142 a b$ & $22.32 \mathrm{c}$ & $22.0 \mathrm{ab}$ & $161.11 \mathrm{ab}$ \\
\hline BG 304 & $0.130 a b$ & $23.00 \mathrm{~b}$ & $22.0 \mathrm{ab}$ & $155.33 \mathrm{~b}$ \\
\hline F-test & ** & ** & ** & ** \\
\hline
\end{tabular}

${ }^{*},{ }^{* *}$ and NS indicate $\mathrm{P}<0.05, \mathrm{P}<0.01$ and not significant, respectively. Means followed by $\mathrm{a}$ common letter are not significantly different at the $5 \%$ level by DMR test.

Analysis of variance showed that root dry weight was higher at the well water compared to drought (Table, 3). Root mass can reflect the status of root growth to a certain extent. Water stress will reduce root dry weight (Ruiz-Lau et al., 2011).Dry weight varied from 7.28 
$\mathrm{g} /$ plant produced by genotype Nerica 4 to $4.20 \mathrm{~g} /$ plant produced by Sakha 102 under well watered, however, root dry weight ranged from $4.47 \mathrm{~g} /$ plant produced by genotype Nerica 4 to $2.63 \mathrm{~g} / \mathrm{plant}$ produced by genotype Sakha 102. Overall, root dry weight were higher in tolerant genotypes than in medium and intolerant genotypes. The genotypes, Nerica 4, Wab 1573 and BG304 had plants that were able to keep water potential high by absorbing water and conducting it to the shoot, due to they posses high values of desirable root traits that associated with drought avoidance mechanism.

Table(3): Influence of well-watered and drought conditions on dry weight of root $(\mathrm{g})$, root- shoot ratio (\%) and specific root length $\left(\mathrm{cm} \mathrm{g}^{-1}\right)$ below 30 $\mathrm{cm}$ from soil surface of rice genotype at maximum tillering stage

\begin{tabular}{|lccc|}
\hline \multicolumn{1}{|c}{ Genotypes } & $\begin{array}{c}\text { Dry weight of } \\
\text { root (g per } \\
\text { plant) }\end{array}$ & $\begin{array}{c}\text { Root- shoot ratio } \\
(\%)\end{array}$ & $\begin{array}{c}\text { Specific root length } \\
\left(\mathbf{m ~ g}^{-1}\right)\end{array}$ \\
\hline Well-watered & & & \\
Sakha 102 & $4.20 \mathrm{~d}$ & $33.20 \mathrm{e}$ & $56 \mathrm{~b}$ \\
Giza 179 & $5.10 \mathrm{c}$ & $35.29 \mathrm{~d}$ & $47 \mathrm{~d}$ \\
Nerica 4 & $7,28 \mathrm{a}$ & $48.44 \mathrm{a}$ & $59 \mathrm{a}$ \\
Wab 1573 & $5.78 \mathrm{~b}$ & $42,53 \mathrm{c}$ & $42 \mathrm{e}$ \\
BG 304 & $5.94 \mathrm{~b}$ & $45.31 \mathrm{~b}$ & $52 \mathrm{C}$ \\
F-test & & & \\
& & & \\
Drought & & & \\
Sakha 102 & $2,63 \mathrm{~d}$ & $23.12 \mathrm{e}$ & $63 \mathrm{~b}$ \\
Giza 179 & $3,50 \mathrm{c}$ & $29.40 \mathrm{~d}$ & $57 \mathrm{c}$ \\
Nerica 4 & $4,47 \mathrm{a}$ & $33.17 \mathrm{a}$ & $62 \mathrm{~b}$ \\
Wab 1573 & $3.93 \mathrm{~b}$ & $32.63 \mathrm{~b}$ & $61 \mathrm{~b}$ \\
BG 304 & $3.99 \mathrm{~b}$ & $30.20 \mathrm{c}$ & $77 \mathrm{a}$ \\
F-test & $* \star$ & $* *$ & a \\
\hline
\end{tabular}

${ }^{*},{ }^{* *}$ and NS indicate $\mathrm{P}<0.05, \mathrm{P}<0.01$ and not significant, respectively. Means followed by $\mathrm{a}$ common letter are not significantly different at the $5 \%$ level by DMR test.

Data presented in (Table, 3) show that root to shoot ratio decreased under drought as reported by (Siopongco et al., 2005). The highest values were recorded by Nerica 4 under well watered and drought conditions.

Concerning, the specific root length, results in (Table, 3) clearly Showed that specific root length increased under drought conditions these results confirm with (Siopongco et al., 2005). The specific root length of all genotypes decreased under well-watered condition than drought condition. Nerica 4 had a higher specific root length under well watered than all genotypes, while BG304 had the highest one under drought condition. 


\section{Heterosis:-}

Per cent heterosis over mid parent and better parents were estimated to know the possible gene action as well as to exploit heterosis for drought associated traits. The magnitude of heterosis manifested over mid parent and better parents are presented. It is evident from (Table, 4$)$ that highly significant estimates of heterosis as a deviation from mid

parent and better parent were exhibited in all studied crosses for growth rate $\left(\mathrm{g}^{\text {day }}{ }^{-1}\right)$ and root length $(\mathrm{cm})$ in $($ Table, 4$)$ under normal and drought conditions. Growth rate $\left(\mathrm{g} \mathrm{day}^{-1}\right)$ and root length $(\mathrm{cm})$ are desirable for drought tolerance in rice. Ganapathy and Ganish (2008).

Cross I (Sakha $102 \times$ Nerica 4) recorded the highest positive heterosis for growth rate $\left(\mathrm{g} \mathrm{day}^{-1}\right)$ the values were $(53.40 \%$ and 39.19 $\%$ under normal and drought, respectively) over mid parent followed whereas range of heterobeltiosis was maximum limit (16.18\%) of the same cross under normal but Cross III (Giza 179xBG 304) was maximum positive heterobeltiosis (6.92) under drought .

Table (4): Heterosis of growth rate $\left(\mathrm{g} \mathrm{day}^{-1}\right)$ and root length $(\mathrm{cm})$ as deviation from mid-parent and better patent under Well-watered and drought conditions

\begin{tabular}{|c|c|c|c|c|c|c|}
\hline \multirow[t]{2}{*}{ Character } & \multirow[t]{2}{*}{ Cross } & \multicolumn{3}{|c|}{ Mean performance } & \multicolumn{2}{|c|}{ Heterosis \% } \\
\hline & & $\mathbf{P 1}^{-}$ & $\mathbf{P 2}^{-}$ & $\mathrm{Fi}^{-}$ & MP & BP \\
\hline \multirow[t]{4}{*}{$\begin{array}{l}\text { Growth rate } \\
\left(\mathrm{g} / \mathrm{day}^{-1}\right)\end{array}$} & $\begin{array}{l}\text { Well- } \\
\text { watered } \\
\text { I } \\
\text { II } \\
\text { III }\end{array}$ & $\begin{array}{l}0.105 \\
0,177 \\
0.148\end{array}$ & $\begin{array}{l}0.204 \\
0.204 \\
0.166\end{array}$ & $\begin{array}{l}0.237 \\
0.215 \\
0.175\end{array}$ & $\begin{array}{l}53.40^{\star *} \\
12.86^{\star *} \\
11.46^{\star *}\end{array}$ & $\begin{array}{c}16.18^{\star \star} \\
5.39^{\star *} \\
5.42^{\star *}\end{array}$ \\
\hline & $\begin{array}{l}\text { L.S.D 5\% } \\
\text { L.S.D 1\% }\end{array}$ & & & & $\begin{array}{l}0.830 \\
0.115 \\
\end{array}$ & $\begin{array}{l}0.05 \\
0.06\end{array}$ \\
\hline & $\begin{array}{l}\text { Drought } \\
\text { I } \\
\text { II } \\
\text { III }\end{array}$ & $\begin{array}{l}0.085 \\
0.142 \\
0.090\end{array}$ & $\begin{array}{l}0.188 \\
0.188 \\
0.130\end{array}$ & $\begin{array}{l}0.190 \\
0.197 \\
0.139\end{array}$ & $\begin{array}{l}39.19^{\star \star} \\
19.39^{\star *} \\
26.36^{\star *}\end{array}$ & $\begin{array}{l}1.06^{\star \star} \\
4.79^{\star \star} \\
6.92^{\star \star}\end{array}$ \\
\hline & $\begin{array}{l}\text { L.S.D 5\% } \\
\text { L.S.D 1\% }\end{array}$ & & & & $\begin{array}{l}0.048 \\
0.066\end{array}$ & $\begin{array}{l}0.005 \\
0.076\end{array}$ \\
\hline \multirow[t]{4}{*}{$\begin{array}{l}\text { Root length } \\
\text { ( cm) }\end{array}$} & $\begin{array}{l}\text { Well- } \\
\text { watered } \\
\text { I } \\
\text { II } \\
\text { III }\end{array}$ & $\begin{array}{l}23.67 \\
24.16 \\
24.00\end{array}$ & $\begin{array}{l}43.67 \\
43.67 \\
31.00\end{array}$ & $\begin{array}{l}45.33 \\
48.0 \\
32.33\end{array}$ & $\begin{array}{l}34.63^{\star *} \\
41.53^{\star *} \\
17.56^{\star *}\end{array}$ & $\begin{array}{l}3.80^{\star \star} \\
9.92^{\star \star} \\
4.29^{\star *}\end{array}$ \\
\hline & $\begin{array}{l}\text { L.S.D 5\% } \\
\text { L.S.D 1\% }\end{array}$ & & & & $\begin{array}{l}1.031 \\
1.431 \\
\end{array}$ & $\begin{array}{l}1.191 \\
1.653 \\
\end{array}$ \\
\hline & $\begin{array}{l}\text { Drought } \\
\text { I } \\
\text { II } \\
\text { III }\end{array}$ & $\begin{array}{l}16.33 \\
22.32 \\
20.00\end{array}$ & $\begin{array}{l}28.13 \\
28.13 \\
23.00\end{array}$ & $\begin{array}{l}29.67 \\
29.33 \\
24.57\end{array}$ & $\begin{array}{l}33.47^{\star *} \\
16.27^{\star *} \\
14.28^{\star *}\end{array}$ & $\begin{array}{l}5.47^{\star \star} \\
4.27^{\star \star} \\
6.83^{\star \star}\end{array}$ \\
\hline & $\begin{array}{l}\text { L.S.D 5\% } \\
\text { L.S.D 1\% }\end{array}$ & & & & $\begin{array}{l}1.292 \\
1.794\end{array}$ & $\begin{array}{l}1.492 \\
2.071\end{array}$ \\
\hline
\end{tabular}


For root length $(\mathrm{cm})$ the cross II (Wab1573xNerica 4) showed the highest per cent of relative heterosis $(41.53 \%)$ under normal condition, while the cross I (Sakha $102 \times$ Nerica 4) recorded the highest value $(33.47 \%)$ under drought condition. While, cross II (Wab1573xNerica 4) showed the highest per cent of heterobeltiosis $(9.92 \%)$ under normal condition, while the cross III (Giza 179xBG 304) recorded the highest value (6.83) under drought condition. 3 hybrids showed significantly positive heterosis and showed this trait heterobeltiosis values were significant and positive

Data presented in (Table, 5) indicated that highly significant positive or negative values of heterosis were recorded for root volume $\left(\mathrm{cm}^{3}\right)$ and number of roots per plant as a deviation from mid-parent in three crosses.

Table (5): Heterosis of root volume $\left(\mathrm{cm}^{3}\right)$ and number of roots / plant as deviation from mid-parent and better patent under Well-watered and drought conditions

\begin{tabular}{|c|c|c|c|c|c|c|}
\hline \multirow[t]{2}{*}{ Character } & \multirow[t]{2}{*}{ Cross } & \multicolumn{3}{|c|}{ Performance } & \multicolumn{2}{|c|}{ Heterosis \% } \\
\hline & & $\mathbf{P 1}^{-}$ & P2 $^{-}$ & $\mathrm{F}^{-}{ }^{-}$ & MP & BP \\
\hline \multirow[t]{4}{*}{$\begin{array}{l}\text { Root volume } \\
\left(\mathrm{cm}^{3}\right)\end{array}$} & $\begin{array}{l}\text { Well-watered } \\
\text { I } \\
\text { II } \\
\text { III }\end{array}$ & $\begin{array}{l}24.67 \\
30.00 \\
25.83\end{array}$ & $\begin{array}{l}37.00 \\
37.00 \\
43.00\end{array}$ & $\begin{array}{l}41.03 \\
29.29 \\
46.96\end{array}$ & $\begin{array}{l}33.06^{\star *} \\
-12.57^{\star *} \\
36.45^{\star *}\end{array}$ & $\begin{array}{c}10.89^{\star \star} \\
-20.84^{\star \star} \\
9.21^{\star \star}\end{array}$ \\
\hline & $\begin{array}{l}\text { L.S.D 5\% } \\
\text { L.S.D } 1 \%\end{array}$ & & & & $\begin{array}{l}1.210 \\
1.680 \\
\end{array}$ & $\begin{array}{l}1.397 \\
1.939 \\
\end{array}$ \\
\hline & $\begin{array}{l}\text { Drought } \\
\text { I } \\
\text { II } \\
\text { III }\end{array}$ & $\begin{array}{l}19.17 \\
22.0 \\
20.0\end{array}$ & $\begin{array}{c}24.53 \\
24.53 \\
22.0\end{array}$ & $\begin{array}{l}32.12 \\
20.04 \\
24.6\end{array}$ & $\begin{array}{c}47.00^{\star \star} \\
-13.86^{\star \star} \\
17.14^{\star *}\end{array}$ & $\begin{array}{c}30.94^{\star \star} \\
-18.30^{\star \star} \\
11.82^{\star \star}\end{array}$ \\
\hline & $\begin{array}{l}\text { L.S.D 5\% } \\
\text { L.S.D } 1 \%\end{array}$ & & & & $\begin{array}{l}2.548 \\
3.537\end{array}$ & $\begin{array}{l}2.942 \\
4.08\end{array}$ \\
\hline \multirow[t]{4}{*}{$\begin{array}{l}\text { No. of roots } \\
\text { per plant }\end{array}$} & $\begin{array}{l}\text { Well-watered } \\
\text { I } \\
\text { II } \\
\text { III }\end{array}$ & $\begin{array}{r}194.0 \\
316.67 \\
200.0\end{array}$ & $\begin{array}{r}356.83 \\
356.83 \\
216.0\end{array}$ & $\begin{array}{l}361.56 \\
364.23 \\
298.1\end{array}$ & $\begin{array}{l}31.28^{\star *} \\
8.16 \\
43.32^{\star \star}\end{array}$ & $\begin{array}{l}1.33 \\
2.07 \\
38.01^{\star \star}\end{array}$ \\
\hline & $\begin{array}{l}\text { L.S.D 5\% } \\
\text { L.S.D 1\% } \\
\end{array}$ & & & & $\begin{array}{l}9.39 \\
13.04 \\
\end{array}$ & $\begin{array}{l}10.85 \\
15.05 \\
\end{array}$ \\
\hline & $\begin{array}{l}\text { Drought } \\
\text { I } \\
\text { II } \\
\text { III }\end{array}$ & $\begin{array}{c}123.0 \\
161.11 \\
140.0\end{array}$ & $\begin{array}{c}170.0 \\
170.0 \\
155.33\end{array}$ & $\begin{array}{l}190.1 \\
188.2 \\
160.2\end{array}$ & $\begin{array}{l}29.76^{* *} \\
13.68^{\star *} \\
8.49^{\star *}\end{array}$ & $\begin{array}{l}11.82 \\
10.71 \\
3.14\end{array}$ \\
\hline & $\begin{array}{l}\text { L.S.D 5\% } \\
\text { L.S.D } 1 \%\end{array}$ & & & & $\begin{array}{l}11.21 \\
15.56\end{array}$ & $\begin{array}{l}12.94 \\
17.97\end{array}$ \\
\hline
\end{tabular}

*, ${ }^{* *}$ Significant at 0.05 and 0.01 levels of probability, respectively.

Crosses, I (Sakha 102xNerica 4), II (Wab1573xNerica 4) and III (Giza 179xBG 304).

These values of mid parent heterosis for root volume $\left(\mathrm{cm}^{3}\right)$ were ranged between $-13.86 \%$ for the cross II (Wab1573xNerica 4) under drought and $47.00 \%$ for cross I (Sakha 102xNerica 4) under drought. 
Also data in (Table, 5) revealed that the heterosis values for root volume $\left(\mathrm{cm}^{3}\right)$ of one cross II (Wab1573xNerica 4) was negative highly significant as deviation from the better parent $(-20.84$ and -18.30 under normal and drought conditions respectively), while the remaining crosses recorded positive significant mean heterotic for this trait.

Also data in (Table, 5) revealed that number of roots per plant is an important character of a hybrid. For this trait the spectrum of variation was from 38.01 cross III (Giza 179xBG 304) to $1.33 \%$ cross I (Sakha 102xNerica 4) for heterobeltiosis, the heterosis values for number of roots per plant of cross III (Giza 179xBG 304) was positive highly significant as deviation from the better parent (38.01), while the remaining crosses recorded non significant mean heterotic for this trait.

Data presented in (Table, 6) indicated that highly significant positive and negative values of heterosis were recorded for dry weight of

root $(\mathrm{g})$ and root- shoot ratio (\%), heterosis for dry weight of root over better parent varied from 12.3 cross I (Sakha 102xNerica 4) to $0.50 \%$ cross III (Giza 179xBG 304) and one cross, was registered negative significant value. With respect to standard heterosis, cross III (Giza 179xBG 304) under normal condition and two hybrids recorded significantly positive value. 
Table(6): Heterosis of dry weight of root $(\mathrm{g})$ and root- shoot ratio (\%) as deviation from mid-parent and better patent crosses under well-watered and drought conditions

\begin{tabular}{|c|c|c|c|c|c|c|}
\hline \multirow[t]{2}{*}{ Character } & \multirow[t]{2}{*}{ Cross } & \multicolumn{3}{|c|}{ Performance } & \multicolumn{2}{|c|}{ Heterosis \% } \\
\hline & & $\mathrm{P1}^{-}$ & $\mathbf{P 2}^{-}$ & $\mathrm{F}^{-}$ & MP & BP \\
\hline \multirow[t]{4}{*}{$\begin{array}{l}\text { Dry weight of } \\
\text { root }(\mathrm{g})\end{array}$} & $\begin{array}{l}\text { Well-watered } \\
\text { I } \\
\text { II } \\
\text { III }\end{array}$ & $\begin{array}{l}4.20 \\
5.78 \\
5.10\end{array}$ & $\begin{array}{l}7,28 \\
7,28 \\
5.94\end{array}$ & $\begin{array}{l}7.29 \\
8.12 \\
5.70\end{array}$ & $\begin{array}{l}27.00^{* *} \\
24.35^{\star *} \\
3.26^{\star *}\end{array}$ & $\begin{array}{l}0.140 \\
11.54^{\star *} \\
-4.04^{\star *}\end{array}$ \\
\hline & $\begin{array}{l}\text { L.S.D 5\% } \\
\text { L.S.D 1\% }\end{array}$ & & & & $\begin{array}{l}0.316 \\
0.439 \\
\end{array}$ & $\begin{array}{l}0.106 \\
0.147 \\
\end{array}$ \\
\hline & $\begin{array}{l}\text { Drought } \\
\text { I } \\
\text { II } \\
\text { III }\end{array}$ & $\begin{array}{l}2,63 \\
3.93 \\
3.50 \\
\end{array}$ & $\begin{array}{l}4,47 \\
4,47 \\
3.99 \\
\end{array}$ & $\begin{array}{l}5.02 \\
4.71 \\
4.01 \\
\end{array}$ & $\begin{array}{l}41.41^{\star *} \\
12.14^{\star \star} \\
7.08^{\star \star}\end{array}$ & $\begin{array}{l}12.3^{\star *} \\
5.37^{\star *} \\
0.50^{\star *}\end{array}$ \\
\hline & $\begin{array}{l}\text { L.S.D 5\% } \\
\text { L.S.D 1\% }\end{array}$ & & & & $\begin{array}{l}0.083 \\
0.115 \\
\end{array}$ & $\begin{array}{l}0.096 \\
0.133 \\
\end{array}$ \\
\hline \multirow[t]{4}{*}{$\begin{array}{c}\text { Root- shoot } \\
\text { ratio (\%) }\end{array}$} & $\begin{array}{l}\text { Well-watered } \\
\text { I } \\
\text { II } \\
\text { III }\end{array}$ & $\begin{array}{l}33.99 \\
42,53 \\
35.29 \\
\end{array}$ & $\begin{array}{l}48.44 \\
48.44 \\
45.31 \\
\end{array}$ & $\begin{array}{l}41.26 \\
51.2 \\
44.63\end{array}$ & $\begin{array}{l}0.11 \\
12.56^{\star *} \\
10.74^{\star *}\end{array}$ & $\begin{array}{l}-14.82^{\star \star} \\
5.70^{\star \star} \\
-1.50^{\star \star}\end{array}$ \\
\hline & $\begin{array}{l}\text { L.S.D 5\% } \\
\text { L.S.D 1\% }\end{array}$ & & & & $\begin{array}{l}0.725 \\
1.006 \\
\end{array}$ & $\begin{array}{l}0.837 \\
1.161 \\
\end{array}$ \\
\hline & $\begin{array}{l}\text { Drought } \\
\text { I } \\
\text { II } \\
\text { III }\end{array}$ & $\begin{array}{l}23.12 \\
32.63 \\
29.40\end{array}$ & $\begin{array}{l}33.17 \\
33.17 \\
30.20 \\
\end{array}$ & $\begin{array}{l}30.5 \\
35.7 \\
31.1\end{array}$ & $\begin{array}{l}8.37^{\star *} \\
8.51^{\star *} \\
4.36^{\star *}\end{array}$ & $\begin{array}{l}-8.05^{\star \star} \\
7.63^{\star \star} \\
2.98^{\star \star}\end{array}$ \\
\hline & $\begin{array}{l}\text { L.S.D 5\% } \\
\text { L.S.D 1\% }\end{array}$ & & & & $\begin{array}{l}0.676 \\
0.938\end{array}$ & $\begin{array}{l}0.781 \\
1.083\end{array}$ \\
\hline
\end{tabular}

${ }^{*},{ }^{* *}$ Significant at 0.05 and 0.01 levels of probability, respectively.

Crosses, I (Sakha 102xNerica 4), II (Wab1573xNerica 4) and III (Giza 179xBG 304).

Cross II (Wab1573xNerica 4) under normal and drought conditions and cross III (Giza 179xBG 304) under drought registered significantly positive heterobeltiosis and cross I (Sakha 102xNerica 4) under normal and drought conditions and cross III (Giza 179xBG 304) under normal recorded significantly negative heterosis under normal condition or root- shoot ratio (\%). The cross I (Sakha $102 x$ Nerica 4) recorded the highest heterobeltiosis and standard heterosis in this regard

Data presented in (Table, 7) indicated that highly significant positive negative values of heterosis were recorded for specific root length as a deviation from mid-parent in three cross. These values of heterosis were ranged between $(-4.84) \%$ for the cross I (Sakha 102x Nerica 4) under drought and $-28.81 \%$ for cross II (Wab1573xNerica 4) under normal condition. Also data in (Table, 7) revealed that the heterosis values for specific root length of one cross I (Sakha $102 x$ Nerica 4) was negative highly significant as deviation from the better parent $(-28.81 \%)$, while the remaining crosses recorded negative significant mean heterotic for this trait except III (Giza 179xBG 304) under normal 
Table (7): Heterosis of specific root length $\left(\mathrm{m} \mathrm{g}^{-1}\right)$ as deviation from midparent and better patent crosses under well-watered and drought conditions

\begin{tabular}{|c|c|c|c|c|c|c|}
\hline \multirow{2}{*}{ Characters } & \multirow[t]{2}{*}{ Crosses } & \multicolumn{3}{|c|}{ Performance } & \multicolumn{2}{|c|}{ Heterosis \% } \\
\hline & & $\mathbf{P} 1^{-}$ & $\mathbf{P 2}^{-}$ & $\mathbf{F} 1^{-}$ & MP & BP \\
\hline \multirow[t]{4}{*}{$\begin{array}{l}\text { Specific root } \\
\text { length }\left(\mathrm{m} \mathrm{g}^{-1}\right)\end{array}$} & $\begin{array}{l}\text { Well-watered } \\
\text { I } \\
\text { II } \\
\text { III }\end{array}$ & $\begin{array}{l}56 \\
42 \\
47\end{array}$ & $\begin{array}{l}59 \\
59 \\
52\end{array}$ & $\begin{array}{l}48 \\
42 \\
57\end{array}$ & $\begin{array}{l}-16.52 \\
-16.83 \\
15.15\end{array}$ & $\begin{array}{l}-18.64 \\
-28.81 \\
9.62\end{array}$ \\
\hline & $\begin{array}{l}\text { L.S.D5 \% } \\
\text { L.S.D1 \% }\end{array}$ & & & & $\begin{array}{l}1.091 \\
1.515 \\
\end{array}$ & $\begin{array}{l}1.261 \\
1.749 \\
\end{array}$ \\
\hline & $\begin{array}{l}\text { Drought } \\
\text { I } \\
\text { II } \\
\text { III }\end{array}$ & $\begin{array}{l}63 \\
61 \\
57\end{array}$ & $\begin{array}{l}62 \\
62 \\
77\end{array}$ & $\begin{array}{l}59 \\
53 \\
61 \\
\end{array}$ & $\begin{array}{l}-5.60 \\
-13.82 \\
-8.96 \\
\end{array}$ & $\begin{array}{l}-4.84 \\
-14.52 \\
-20.78 \\
\end{array}$ \\
\hline & $\begin{array}{l}\text { L.S.D 5\% } \\
\text { L.S.D 1\% }\end{array}$ & & & & $\begin{array}{l}0.967 \\
1.342\end{array}$ & $\begin{array}{l}1.117 \\
1.550\end{array}$ \\
\hline
\end{tabular}

* ${ }^{* *}$ Significant at 0.05 and 0.01 levels of probability, respectively.

Crosses,I (Sakha 102xNerica 4), II (Wab1573xNerica 4) and III (Giza 179xBG 304)

More or less, fifty per cent of the hybrids exhibited desirable significant heterosis over mid parent for all root characters under study and recorded significantly positive better parent heterosis indicating different role of these traits in drought tolerance mechanism of parents and their inheritance in the hybrids. Root length and root dry weight are desired for a genotype to be resistant to drought as revealed by the earlier workers (Michael, and Rangasamy, 2002) and (Anbumalarmathi, 2005). And a lot of hybrid for specific root length exhibited significantly negative heterosis over better parents, this hybrids also utilized for future breeding program for development of drought tolerance lines Thus parents producing non-heterotic hybrids for leaf drying and drought recovery rate may be preferred while aiming to produce drought tolerance hybrids.

From the obtained results, it was clear that significant and highly significant and positive of estimated heterosis as a deviation from mid parent and better parent were obtained for root length, root volume, number of root per plant and root shoot ratio ,while similar results were reported by Abed El -Alattef et al (2008).Ganapathy and Ganish (2008) and Hassan (2011) the most desirable cross for all root characters under study was that cross III and cross I.

\section{Phenotypic correlation coefficients:}

The study of relationships among morphological of roots characters is great importance. The estimates of correlation coefficient among all studied characters are presented in (Table, 8 ) 
Table 8: Estimates of phenotypic correlation coefficients among all cultivars of studied characters

\begin{tabular}{|c|c|c|c|c|c|c|c|}
\hline characters & 1 & 2 & $\overline{3}$ & $\overline{4}$ & $\overline{5}$ & 6 & 7 \\
\hline $\begin{array}{l}1-\text { Root growth rate } \\
N \\
\text { D } \\
\end{array}$ & $\begin{array}{l}1.0 \\
1.0\end{array}$ & & & & & & \\
\hline $\begin{array}{l}2-\text { Root length }(\mathrm{cm}) \\
N \\
\text { D }\end{array}$ & $\begin{array}{l}.694 \\
.750^{*}\end{array}$ & $\begin{array}{l}1.0 \\
1.0\end{array}$ & & & & & \\
\hline $\begin{array}{l}3-\text { Root volume }\left(\mathrm{cm}^{3}\right) \\
\text { N } \\
\text { D }\end{array}$ & $\begin{array}{l}328 \\
.474 \\
\end{array}$ & $\begin{array}{l}.055 \\
.626\end{array}$ & $\begin{array}{l}1.0 \\
1.0\end{array}$ & & & & \\
\hline $\begin{array}{l}\text { 4- No. of roots/plant } \\
\text { N } \\
\text { D }\end{array}$ & $\begin{array}{l}898^{* *} \\
.865\end{array}$ & $\begin{array}{l}.714^{*} \\
.972^{* *}\end{array}$ & $\begin{array}{l}.137 \\
.619\end{array}$ & $\begin{array}{l}1.0 \\
1.0\end{array}$ & & & \\
\hline $\begin{array}{l}\text { 5-Dry weight of root } \\
\text { (g)per plant } \\
N \\
\text { D }\end{array}$ & $\begin{array}{l}.925^{* *} \\
.785\end{array}$ & $\begin{array}{l}.815^{*} \\
.978^{* *}\end{array}$ & $\begin{array}{l}.130 \\
.674\end{array}$ & $\begin{array}{l}870^{*} \\
.981^{*}\end{array}$ & $\begin{array}{l}1.0 \\
1.0\end{array}$ & & \\
\hline $\begin{array}{l}\text { 6-Root-shoot ratio(\%) } \\
N \\
\text { D }\end{array}$ & $\begin{array}{l}.710 * \\
.612\end{array}$ & $\begin{array}{l}588 \\
.809^{*}\end{array}$ & $\begin{array}{l}.272 \\
185\end{array}$ & $\begin{array}{l}.728^{*} \\
.815^{* *}\end{array}$ & $\begin{array}{l}.838^{* *} \\
.814^{*}\end{array}$ & $\begin{array}{l}1.0 \\
1.0\end{array}$ & \\
\hline $\begin{array}{l}\text { 7-Specific root length } \\
\left(\mathrm{m} \mathrm{g}^{-1}\right) \\
N \\
D\end{array}$ & $\begin{array}{l}-.295 \\
-.305\end{array}$ & $\begin{array}{c}073 \\
-.285\end{array}$ & $\begin{array}{c}181 \\
-.028\end{array}$ & $\begin{array}{l}-.215- \\
-.319\end{array}$ & $\begin{array}{l}-.291 \\
-.228\end{array}$ & $\begin{array}{l}-.084 \\
-.308-\end{array}$ & $\begin{array}{l}1.0 \\
1.0\end{array}$ \\
\hline
\end{tabular}

*, ** Significant at 0.05 and 0.01 levels of probability, respectively.

Regarding to correlations between root growth rate and all other studied traits, root growth rate was highly significantly and positively correlated root length $(\mathrm{cm})$ under drought condition, number of roots/plant, dry weight of root $(\mathrm{g})$ per plant under drought and well water conditions and root-shoot ratio(\%) under normal condition under study.

As for, root length it showed highly significant positive correlation coefficient root length is Significant with number of roots/plant and dry weight of root $(\mathrm{g})$ per plant under drought and well water conditions and root-shoot ratio(\%) under drought condition

Concerning number root volume $\left(\mathrm{cm}^{3}\right)$, data showed Root volume $\left(\mathrm{cm}^{3}\right)$ is no Significant with all characters under study.

For number of roots/plant, results showed that highly significant positive correlation coefficient between this trait with dry weight of root (g) per plant under drought and well water conditions and root-shoot ratio (\%) under normal condition.

As far as dry weight of root (g) per plant was concerned, positive significant and highly significant correlation coefficient estimates were found between this trait and root-shoot ratio (\%) under normal and drought condition

Finally, specific root length $\left(\mathrm{m} \mathrm{g}^{-1}\right)$ is no significant with all characters under study 


\section{CONCLUSION}

The rice genotypes evaluated differ in root characters under study and respond differently to well water and drought. The tolerance genotype had roots characters which produce increased tolerant drought conditions, can increase water reserves and can use heterosis to improvement the tolerance.

\section{REFERENCES}

AbdAllah ,A.A.; Shimaa A.Badawy; B.A Zayed and A.A. El.Gohary (2010).The Role of Root System Traits in the Drought Tolerance of Rice (Oryza sativa L.) World Academy of Science, Engineering and Technology 44 - 1388-1392.

Abed El- Lattef, A.S.M.; A.B. El Abed; A.A Madyan and W.M.H. ElKhouby (2008).Inheritance of earliness, grain quality and some grain quality traits in rice (Oryza sativa L.) under water deficiency conditions .J.Agric.Res. Kaferelshiekh Univ., 34:9931019.

Anbumalarmathi, J. (2005). Genetic analysis for drought tolerance and yield component traits in rice (Oryza sativa L.), Ph.D. Thesis, TNAU, Coimbatore, India.

Bernier, J.; G. N. Atlin; R.Serraj; A. Kumar and Spaner, D. (2008).Breeding upland rice for drought resistance. Journal of the Science of Food and Agriculture, v.88, p.927-939.

Beyrouty,C.A.(2002)."Eco physiology of roots of aquatic plants, "in The Half,3rdEdn,edsY.Waisel,A.Eshel,andU.Kafkafi(New York, NY: Marcel Dekker ,Inc.),1007-1024.

Cleber M. G; L.F. Stone; P. H. N. Rangel and Ana C. de L. Silva (2013) Tolerance of upland rice genotypes to water deficit. Revisita Brasillera de Engenharia Agricola Ambiental. v.17, n.8, p.805-810.

Duncan, D.B. (1955). Multiple range and multiple F. Test .Biometrics, 11:1-24.

Dewey,D.R. and K.H.Lu (1959). A correlation and Path - coefficient analysis of components of crested wheatgrass seed production .Agron. J., 51: 515-518.

Fageria,N.K., and A.Moreira (2011).The role of mineral nutrition on root crop growth of crop plants. Adv. Agron. 80, 63-152.

Fitter, A.H. (1991). "Characteristics and functions of root systems". In: Y. Waisel, A. Eshel and U. kafkafi eds., Plant Roots- the Hidden Half.Marcel Dekker, New York. 3-25.

Ganapathy,S. and S.K. Ganesg(2008).Heterosis analysis for physio-

Morphological in relation to drought tolerance in rice (Oryza sativa L.).World J.Agri.Sci., 5:623-629.

Gomez KA and Gomez AA (1984). Statistical Procedures for agricultural Research, $2^{\text {nd }}$ edition.;680

Gowda,V.R.P.; A.Henry; A.Yamauchi; H.E.Shashidhar and Serraj,R 
(2011). Oat biology and genetic improvement for drought avoid anceinrice. FieldCrops Res. 122, 1-13.

Harovon Mogel,K.(2013).Interactions key to beating future droughts. CSA News 58, 4-9.doi:10.2134/csa2013-58-2-1.

Hassan,H. M.; A. B.El-Abed and N.M. El-Baghdady.(2011). Combining ability for some root .physiological and grain quality traits in rice (Oryza sative L.)under water deficit conditions J. Agric. Res. Kafrelshiekh Univ., 37:239-256.

Ingram, K.T.; F.D. Bueno; O.S.Namuco; E.B. Yambao and C.A. Beyrouty,(1994)." Rice root traits for drought resistance and their genetic variation". IRRI, Philippines. In G.J.D. Kirk ed., Rice Roots. Nutrient and Water Use.67-77.

Intergovernmental Panel on Climate Change (IPCC).(2012). "Summary for Policy makers," in Managing the Risks of Extreme Events and Disasters to Advance Climate Change Adaptation. A Special Report of Working Groups I and II of the Intergovernmental Panel on Climate Change, eds C.B. Field ,V.Barros, T.F.Stocker, D.Qin,D.J.Dokken,K.L.Ebi,etal. Cambridge: Cambridge University Press), 1-19.

Kato Y. and M. Okami. (2011).Root morphology, hydraulic conductivity and plant water relations of high-yielding rice grown under aerobic conditions. Annals of Botany 108: 575-583.

Kondo, M.; P.P. Pablico; D.V. Aragones; R. Agbisit; J. Abe and S .Morita. (2003).Genotypic and environmental variations in root morphology in rice genotypes under upland field conditions. PlantSoil 255, 189-200.

Mather K. and J.L.Jinks (1971). Biometrical Genetics. Cornell University Press. Ithaca, New York, Pages: 231.

Michael, G.S. and P. Rangasamy .(2002). Correlation and path analysis of yield and physiological characters in drought resistant rice (Oryza sativa L.). Int. J. Mendel., 19(1-2): 33-34.

Passioura,J.B.(2012).Phenotyping for drought tolerance in grain crops: when is it useful to breeders .Funct.Plant Biol. 39, 851.doi:10.1071/FP12079

Ruiz-Lau, N.; F. Medina-Lara; Y. Minero-García; E. ZamudioMoreno; A. Guzmán-Antonio and I. Echevarría-Machado .(2011) Water deficit affects the accumulation of capsaicinoids in fruits of Capsicum chinense J acq. Hort Science 46:487-492.

Sestak , Z.; J. Catsky and P.G.Jarvis (1971). Plant photosynthetic production, Manual of Methods, Dr. W. Junk, N.V. Publishers, The Hague, 818p

Siopongco J. D..; A. Yamauchi; H. Salekdeh; J. Bennett and L. J. Wade (2005).Root Growth and Water Extraction Response of DoubledHaploid Rice Lines to Drought and Rewatering during the Vegetative Stage. Plant Prod. Sci. 8(5): 497 - 508. 


\section{تقييم تحمل الجفاف وقوه الهجين لبعض صفات الجذور في الأرز}

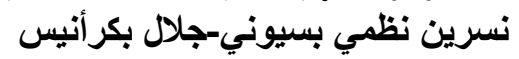

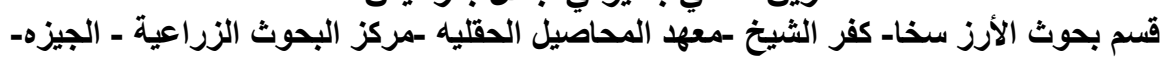
مصر

أقيمت تجربتان حقليتان بالمزرعة البحثيه لمركز البحوث و التدريب في الأرز

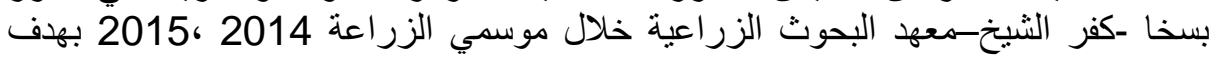

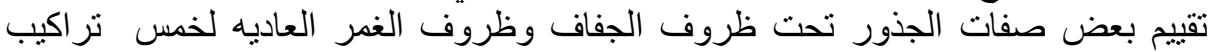

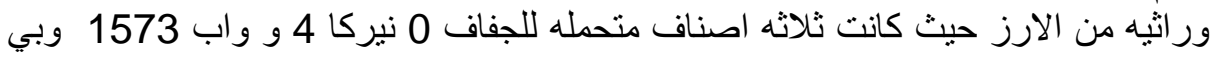

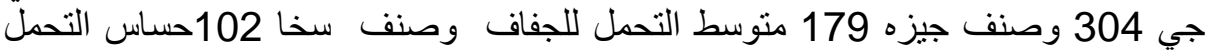

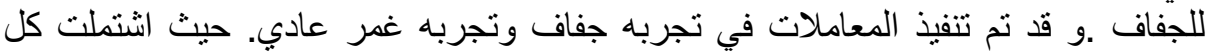

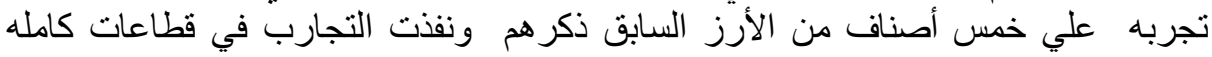

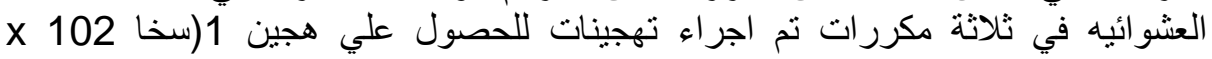

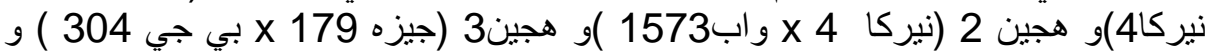

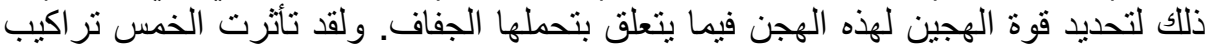

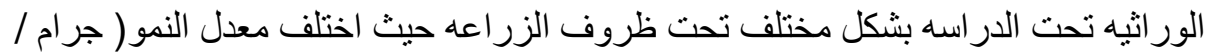

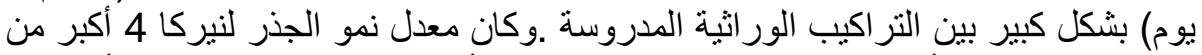

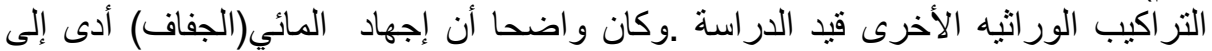

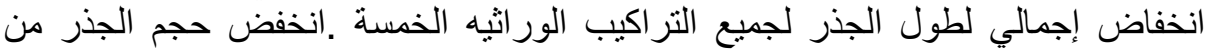

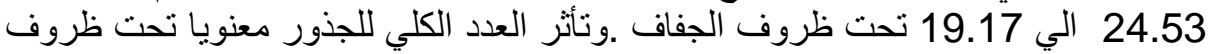

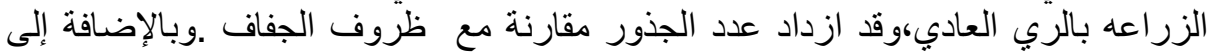

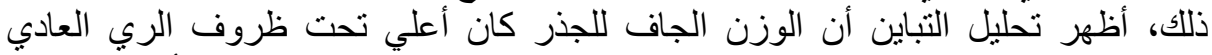

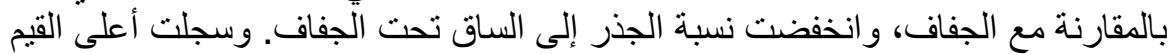

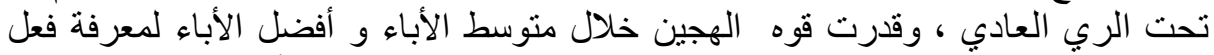

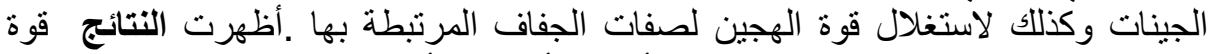

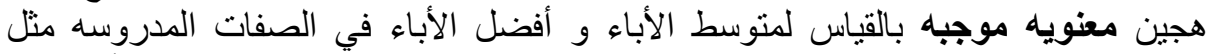

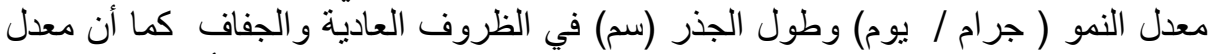
النمو ( جرام / يوم) وطول الجذر (سم) تكون مرغوبة لتحمل الجن الجفاف في الأرز. 